\title{
Newsvendor Model Of Capacity Sharing
}

\author{
R. Berry, M. Honig, T. Nguyen, V. \\ Subramanian, H. Zhou \\ EECS Department \\ Northwestern University \\ Evanston, IL 60208 \\ \{rberry, mh, thanh, vjsubram, \\ hang.zhou\}@eecs.northwestern.edu
}

\author{
R. Vohra \\ CMS-EMS, Kellogg School of Management \\ Northwestern University \\ Evanston, IL 60208 \\ r-vohra@kellogg.northwestern.edu
}

\section{INTRODUCTION}

Capacity sharing in the form of roaming agreements have long been a fixture of cellular service. Historically, the main driver behind roaming agreements is to extend the coverage of a wireless carrier's network into regions where that carrier has no infrastructure, thus making the service more attractive to customers who "roam" into a new region. Here we focus on a different form of capacity sharing, namely acquiring "overflow" capacity from another provider during periods of high demand. Such sharing may provide carriers with an attractive means to better meet their rapidly increasing bandwidth demands. On the other hand, the presence of such a sharing agreement could encourage providers to under-invest in their networks, resulting in poorer performance. We consider a stylized model of such a situation to gain insight into these trade-offs. Specifically, we adapt the newsvendor model from operation management [8] to this situation. The newsvendor model applies to a single firm that is determining how much inventory to stock in the face of uncertain demand. Here, we consider two wireless carriers, who are determining how much capacity to invest in, also in the face of uncertain demand. Without any capacity sharing, this reduces to the standard newsvendor model for each carrier. However, with sharing, the carriers's investment decisions become coupled since the revenue one carrier earns form sharing capacity with another depends on the other's capacity investment. We model this interaction as a game and give conditions for when the game has a unique pure strategy Nash equilibrium. Our analysis applies for two different sharing models one in which a carrier first serves its own customers before allocating an excess capacity to the other provider's customers and one in which carriers do not discriminate between their own traffic and the other carrier's. Numerical comparisons of the resulting equilibria are also given for different demand distributions.

In terms of related work, there has been a line of literature studying how carriers set prices in roaming agreements (see e.g. [4]). Much of this work focuses on a known demand and

Copyright is held by author/owner(s).

seeks to understand if firms pricing decision lead to collusive behavior. An extension of this line of work that also considers investment can be found in [9]. Here we assume that prices for roaming are given exogenously and focus instead on the carriers investment decisions in the face of uncertain demand. There has also been increasing interest in sharing "raw spectrum" between different providers (e.g. [1]). This differs from the capacity sharing model considered here in that if a carrier receives raw spectrum from another carrier, it would still have to use its own infrastructure to utilize this spectrum. Finally, we note that the type of capacity sharing model considered here may be applicable to other settings as well, such as sharing between providers of renewable energy in real-time electricity markets [6].

\section{NON-SHARING MODEL}

We consider a situation with two carriers, 1 and 2, who are faced with unknown demands in each of their markets. They each procure capacity/spectrum as in the newsvendor model [8]. The initial scenario assumes that there is no interaction between the two carriers. We denote the reward per unit demand of the carriers by $p_{i}$ and the cost per unit capacity by $c_{i}$ for $i=1,2$. The demands are denoted by a non-negative stochastic quantity $D_{i}$ with probability density function $f_{i}(\cdot)$ and cumulative distribution function $F_{i}(\cdot)$ (complementary cumulative distribution function being $\left.F_{i}^{C}(\cdot)\right)$. Initially we will consider independent demands.

The carriers chose to buy capacity $q_{i}$ at the beginning. Then the expected profit of each carrier $\tilde{\pi}_{i}$ is given

$$
\begin{aligned}
\mathbb{E}\left[\tilde{\pi}_{i}\right] & =p_{i} \mathbb{E}\left[\min \left(q_{i}, D_{i}\right)\right]-c_{i} q_{i} \\
& =p_{i} q_{i} F_{i}^{C}\left(q_{i}\right)+p_{i} \int_{0}^{q_{i}} x f_{i}(x) d x-c_{i} q_{i}
\end{aligned}
$$

Since $\min \left(q_{i}, x\right)=: q_{i} \wedge x$ is a concave function of $q_{i}$ for any $x \in \mathbb{R}$, it follows that $p_{i} \mathbb{E}\left[\min \left(q_{i}, D_{i}\right)\right]$ is also a concave function of $q_{i}$. If, in addition, a density exists for $F_{i}(\cdot)$, then by taking derivatives, it is easy to see that the expected profit is a strictly concave function of $q_{i}$ and the optimal amount of capacity purchased is given by

$$
q_{i}^{*}=F_{i}^{-1}\left(1-\frac{c_{i}}{p_{i}}\right)
$$


where $F_{i}^{-1}(\cdot)$ are inverse functions given by

$$
F_{i}^{-1}(y)=\inf \left\{x: F_{i}(x)=y\right\}
$$

This will be the base-line comparison scenario for our analysis, in other words, the outside option available to the carriers. Note that the analysis carries over unchanged even when the demands are dependent random variables with the marginal distributions having densities.

\section{SHARING MODEL A}

We now allow the carriers to share their excess spectrum, i.e., if one of them has excess demand and the other excess spectrum, then the excess spectrum can be applied to the excess demand with the spectrum holder charging for usage of her spectrum. For explaining the capacity/spectrum sharing scheme let us assume the instantiation of the demands is such that $q_{2}<D_{2}$ and $q_{1} \geq D_{1}$ so that carrier 1 has excess spectrum that can be used to serve the excess demand that carrier 2 has. Assume that carrier 1 charges a price $c_{r}$ per unit of capacity. Then she makes an additional profit of $c_{r} \min \left(D_{2}-q_{2}, q_{1}-D_{1}\right)$ while carrier 2 makes an additional profit of $\left(p_{2}-c_{r}\right) \min \left(D_{2}-q_{2}, q_{1}-D_{1}\right)$. Here we assume that the carriers can distinguish the different types of traffic and serve all the native demand before serving the traffic of the other carrier. We will assume that $c_{r} \leq p_{2}$, else the expected profit of carrier 2 will decrease, reducing her incentive to serve the extra demand. With such an assumption in place we can consider the maximum amount of revenue that can be obtained from the transaction to be $p_{2} \min \left(D_{2}-q_{2}, q_{1}-D_{1}\right)$, and this has to be shared between the carriers in the some fashion. We will assume in the current scenario carrier 2 gets an $\beta \in[0,1]$ fraction of this revenue with the rest going to carrier 1 . In the opposite scenario where carrier 2 has the excess spectrum and carrier 1 the excess demand, we will assume that carrier 2 gets a $1-\alpha$ fraction of the revenue, which equals $p_{1} \min \left(D_{1}-q_{1}, q_{2}-D_{2}\right)$, with carrier 2 retaining the remainder of the money. An $\beta$ (or $\alpha$ ) less than a half implies that the owner of the spectrum keeps more of the money to be made from the sharing contract, and the vice-versa for the case of $\beta$ greater than a half. This will be an important distinction in the analysis later on.

Unlike in the non-sharing case, we now have a game that the two carriers play. They will try to choose their purchases of spectrum $q_{i}$ using the knowledge of the distributions of the demands of both carriers and the sharing mechanism in place. The timing of the game is the following: first the sharing contract is fixed, i.e., the $\alpha$ and $\beta$ parameters are given; next the carriers purchase capacity/spectrum with the knowledge of the contract and the distribution of the demands; and finally, the payoffs are revealed once the demands are revealed. In such a setting we will explore the existence of pure Bayes-Nash equilibria [2]. As mentioned before, if one includes the bigger question of whether carriers will be incentivized to sharing spectrum, then one must include the constraint that their profits are above what they can obtain in the no-sharing scenario. This will be discussed further in the analysis of the resulting equilibria. Additionally, though we present results assuming that the demands are independent, the analysis carries through unchanged when the demands have a joint probability function with a density.

The expected profit of carrier 2 under this setting when she purchases $q_{2}$ and carrier 1 purchases $q_{1}$ is then given by

$$
\begin{aligned}
& \mathbb{E}\left[\pi_{2}\right]=p_{2} q_{2} F_{2}^{C}\left(q_{2}\right)+p_{2} \int_{0}^{q_{2}} x f_{2}(x) d x-c_{2} q_{2} \\
& +\beta p_{2} \int_{q_{2}}^{\infty} \int_{0}^{q_{1}} \min \left(y-q_{2}, q_{1}-x\right) f_{1}(x) f_{2}(y) d y d x \\
& +(1-\alpha) p_{1} \int_{0}^{q_{2}} \int_{q_{1}}^{\infty} \min \left(q_{2}-y, x-q_{1}\right) f_{1}(x) f_{2}(y) d y d x
\end{aligned}
$$

The expected profit for carrier 1 can be written in a similar manner. The analysis proceeds by determining the bestresponse of carrier 2 when carrier 1 purchases spectrum $q_{1}$, and vice-versa. We will rule out the cases $(\alpha=1, \beta=0)$ and $(\alpha=0, \beta=1)$ where one carrier gets all the profit from sharing. In these cases, one carrier purchases as per the nosharing scenario and the other carrier purchases spectrum taking into account this action. The main result is then the following.

TheOREM 1. The spectrum game outlined above has a unique pure Nash equilibrium if $p_{1} \geq(1-\beta) p_{2}$ and $p_{2} \geq$ $(1-\alpha) p_{1}$. In addition, the equilibrium can be obtained by iterating the best-response correspondences.

Proof. The proof is carried out in two steps. The first step shows that the profit maximization problem of each carrier is a convex optimization problem with a unique solution. This then establishes the continuity of each carrier's best response in the choice made by the other carrier. We also show that it suffices to consider strategies in a compact and connected set. Therefore, existence of pure Nash equilibria follows using Brouwer's fixed point theorem [3]. In the second step we prove that iterating the best-response correspondences results in a contraction, which then establishes the uniqueness of the pure Nash equilibrium via the Banach fixed point theorem [3]. $\diamond$

An important sub-case that we will discuss further is when $p_{1}=p_{2}=p$, i.e., the carriers receive the same per unit demand payoff. This can be justified by assuming that the service provided by the carriers is the same. Under this assumption it is sufficient to consider the cost of spectrum normalized by $p$. If we further assume that $\alpha+\beta=1$, then we get a simpler alternative analysis. In this case it can be verified that we have a potential game [5]; using the results of [5] can also be obtained.

We would like to compare the Nash equilibrium with the jointly optimal spectrum purchase that tries to maximize the sum of the expected profits of both the carriers. This is not necessarily the objective of the social planner who would instead like to maximize the total demand served by 
the carriers subject to the providers preferring sharing to not sharing. Specializing to the case of $p_{1}=p_{2}=p$, we have a convex optimization to solve that yields the following solution: the carrier with the lower cost per unit of spectrum buys spectrum facing the total demand while the other carrier stays out. If the costs are the same, then any partition of the spectrum between the carriers is acceptable.

\section{SHARING MODEL B}

We consider an alternate sharing model where the carrier accepting traffic from its competitor cannot discriminate between the two types of traffic that it has to carry owing to sharing. Once again we will start with the independent markets model and the game between the carriers is to decide on the spectrum investment level so as to maximize individual profit. For simplicity we will assume that the carrier with excess demand transfers the revenue from the amount of traffic served by the carrier with the excess spectrum. The carrier who faces the types of demands serves each in proportion to the submitted demand when the total is above the spectrum investment. Going into further details the expected profit of carrier 1 is given by

$\mathbb{E}\left[\pi_{1}\right]=-c_{1} q_{1}+p_{1} q_{1} F_{1}^{C}\left(q_{1}\right)+p_{1} F_{2}\left(q_{2}\right) \int_{0}^{q_{1}} x f_{1}(x) d x+$
$\int_{q_{2}}^{\infty} \int_{0}^{q_{1}} \min \left(\frac{q_{1}}{x+y-q_{2}}, 1\right)\left(p_{1} x+p_{2}\left(y-q_{2}\right)\right) f_{1}(x) f_{2}(y) d x d y$

Note that there is no sharing when both carriers either have excess spectrum or excess demand. Additionally, when carrier 1 has excess demand, then she serves $q_{1}$ amount of traffic and sends the remainder to carrier 2, who then implements the proportional sharing rule. In this setting the two carriers try to cooperate as much as possible. Again the case of $p_{1}=p_{2}=p$ is easily amenable to analysis; it is possible to construct distributions for which one does not get the concavity properties used in the proof of Theorem 1 when $p_{1}$ and $p_{2}$ are distinct. Here we get a further simplification to

$$
\begin{aligned}
& \mathbb{E}\left[\pi_{1}\right]=\left(p-c_{1}\right) q_{1}-p F_{2}\left(q_{2}\right) \int_{0}^{q_{1}}\left(q_{1}-x\right) f_{1}(x) d x \\
& -p \int_{q_{2}}^{q_{1}+q_{2}} \int_{0}^{q_{1}+q_{2}-y}\left(q_{1}+q_{2}-x-y\right) f_{1}(x) f_{2}(y) d x d y
\end{aligned}
$$

With some algebraic modifications, it is possible to show that the expected profit of carrier $i$ in $q_{i}$ in this model is exactly the same as that of model A with $\alpha=\beta=0$, i.e., the regime where the carriers that own the spectrum keep the profit from sharing spectrum. Therefore, in both cases the same amount spectrum will be purchased by both carriers.

\section{DISCUSSION}

In this section we will explore the consequences of Theorem 1 and compare the resulting equilibrium with the nosharing scenario; a finer analysis of Model B is for future research. For simplicity we will restrict attention to a symmetric setting where the marginal distributions, the per unit cost of spectrum and the sharing contracts are the same for both service providers; the normalized per unit cost of spectrum will be assumed to be 0.5 in the remainder. We will vary the dependence structure between the two markets. Intuitively it is clear that there will be minimal benefits to sharing if the markets are co-monotone, i.e., perfectly dependent which in this case corresponds to the same random variable being chosen for both. On the contrary, countermonotonicity would yield the most benefits as the probability of having excess demand at one service provider and excess spectrum supply at the other provider is maximized. Given the technical requirements of having a joint density function, we will approach these two extreme cases via the parameterized Frank copulas [7]; for co-monotone the parameter choice is 50 , while it is -50 for the counter monotone case. This also allows us to consider the case when the markets are independent where the parameter choice is 0 . The approximations to the co-monotone and counter monotone copulas will be close to the optimal solution. However, the numerical solution will not capture certain specific behaviors, e.g., for the symmetric co-monotone case, it is easy to see that there is no advantage to sharing and the newsvendor optimal solution is also the optimal purchase with any of the sharing contracts of Model A.

We will consider three types of demands starting from distributions with bounded to heavy-tailed distributions. In all cases we assume that the mean demand is 1 and the marginal distributions are the same. We compare the nosharing spectrum purchase to the Nash equilibrium purchase for symmetric contracts as the contract sharing parameters $\alpha$ and $\beta$ are varied from 0 to 1 . The specific demand distributions that we consider are the following: uniform $[0,2]$ random variables; log-normal random variables with mean parameter -2 and variance parameter 2 ; and pareto random variables with parameters $0.49495,0.5$ and 0.01 so that the minimum value is 0.01 . Note that we start with a distribution with bounded support. Then we consider a random variable where all moments are finite but the moment generating function is not. Finally we consider a random variable that has infinite variance. This allows to explore a widerange of distributional behaviors.

First we assume the demands follow a lognormal distribution. Using the news-vendor model from Section 2, the optimal spectrum purchase is $q_{n s}^{*} \approx 0.1353$ units. In Figure 1 we compare the resulting Nash equilibrium spectrum purchase for symmetric contracts as the contract sharing parameters $\alpha$ and $\beta$ are varied from 0 to 1 . In all cases, the expected profit is greater than the no sharing profit so that the carriers will participate in the contract. In general, if the spectrum owner keeps most of the revenue from sharing $(\alpha, \beta<0.5)$, then more spectrum is bought. In addition, when the demands are independent or counter monotone even when the spectrum owner keeps a smaller part of the revenue from sharing, there is greater incentive to purchase more spectrum than in the no sharing case. The per car- 


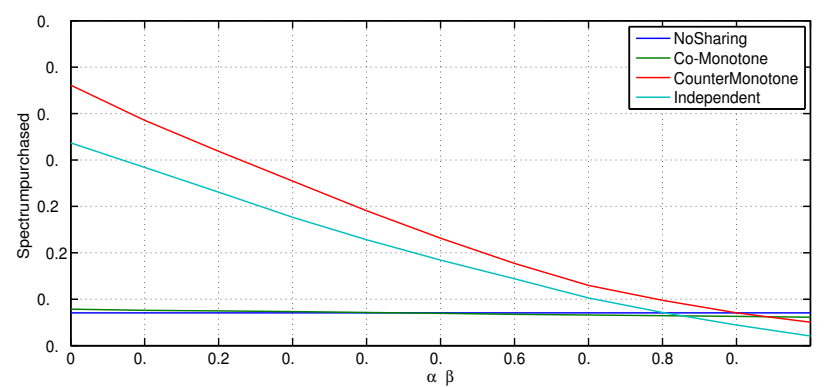

Figure 1: Comparison of Nash equilibrium to the no sharing scenario for lognormal demand.

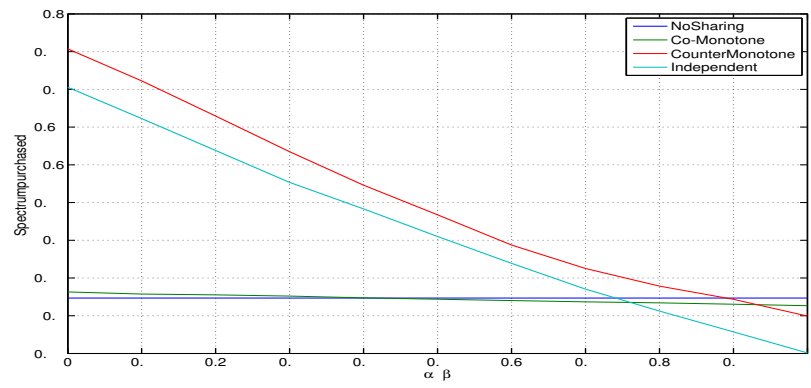

Figure 2: Comparison of Nash equilibrium to the no sharing scenario for pareto demand.

rier spectrum purchase for the sum profit maximization is $0.2784,0.2508$ and 0.1353 for the counter monotone, independent and co-monotone cases, respectively. Therefore, if the spectrum owner gets the bulk of the revenue from sharing, then there is incentive to buy more spectrum than the sum profit maximization strategy.

First we assume the demands follow a pareto distribution. The optimal spectrum purchase in the no sharing case is $q_{n s}^{*} \approx 0.4234$ units. In Figure 2 we compare the resulting Nash equilibrium spectrum purchase for symmetric contracts as the contract sharing parameters $\alpha$ and $\beta$ are varied from 0 to 1 . In all cases, the expected profit is greater than the no sharing profit so that the carriers will participate in the contract. The per carrier spectrum purchase for the sum profit maximization is $0.5867,0.5756$ and 0.4229 for the counter monotone, independent and co-monotone cases, respectively. Again, if the spectrum owner gets the bulk of the revenue from sharing, then there is incentive to buy more spectrum than the sum profit maximization strategy.

Finally we consider the case of the demands being uniformly distributed in $[0,2]$. In this case, the service providers each purchase 1 unit of spectrum when there is no sharing. Again, the expected profit from sharing is strictly better than the no sharing case. From Figure 3 it is clear that the carriers buy more spectrum only when the spectrum owner gets a bigger share of the revenue from sharing. The sum profit maximizing strategy for all cases would be for both carriers to buy 1 unit of spectrum.

The broad conclusions that we can draw from the nu-

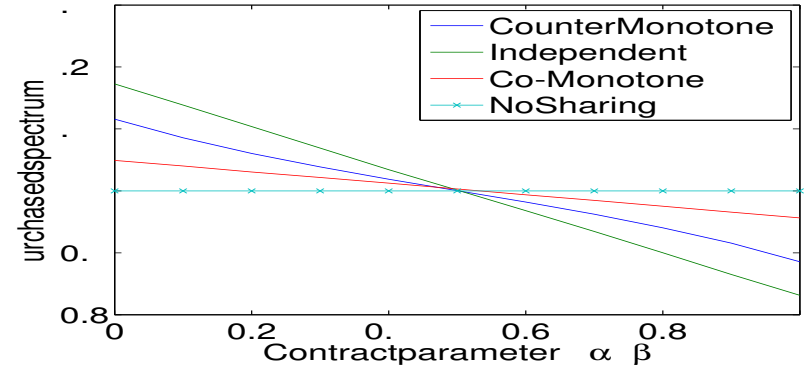

Figure 3: Comparison of Nash equilibrium to the no sharing scenario for uniform demand.

merical investigations is that sharing increases the expected profit for the carriers so that it is in their best interests to do so. We believe this holds in general and not just for the examples considered above. The specific structure of the contracts determines whether more or less spectrum is purchased with sharing when compared to the no sharing case. In general, if the spectrum owner retains the bulk of the revenue from sharing, then more spectrum is purchased. Finally, greater benefits are obtained from sharing when the distributions are heavy-tailed and the joint distributions counter monotone.

\section{ACKNOWLEDGEMENTS}

This research was supported in part by NSF under grant CNS-0905407.

\section{REFERENCES}

[1] R. Berry, M. Honig, and R. Vohra. Spectrum markets: motivation, challenges and implications. IEEE Communications Magazine, 48(11):146-155, 2010.

[2] D. Fudenberg and J. Tirole. Game theory. MIT Press, Cambridge, MA, 1991.

[3] A. Granas and J. Dugundji. Fixed point theory. Springer Monographs in Mathematics. Springer-Verlag, New York, 2003.

[4] J.-J. Laffont and J. Tirole. Competition in Telecommunications. MIT Press, Cambridge, MA, 1998.

[5] D. Monderer and L. S. Shapley. Potential games. Games Econom. Behav., 14(1):124-143, 1996.

[6] J. Nair, S. Adlakha, and A. Wierman. Energy procurement strategies in the presence of intermittent sources. 2012. Preprint.

[7] R. B. Nelsen. An introduction to copulas. Springer Series in Statistics. Springer, New York, $2^{\text {nd }}$ edition, 2006.

[8] W. J. Stevenson. Operations Management. Irwin/McGraw-Hill, $11^{\text {th }}$ edition, 2011.

[9] T. Vallett and C. Cambini. Investment and network competition. The RAND Journal of Economics, 36(2):446-467, 2005. 